Baltic Astronomy, vol.24, 137-143, 2015

\title{
DETECTION OF UNRESOLVED BINARIES WITH MULTICOLOR PHOTOMETRY
}

D. Chulkov ${ }^{1}$, M. Prokhorov ${ }^{2}$, O. Malkov ${ }^{1}$, S. Sichevskij ${ }^{1}$, N. Krussanova ${ }^{2}$,

A. Mironov ${ }^{2}$, A. Zakharov ${ }^{2}$ and A. Kniazev ${ }^{2,3,4}$

1 Institute of Astronomy of the Russian Academy of Sciences, Pyatnitskaya St. 48, Moscow 119017, Russia; chulkov@inasan.ru

2 Sternberg Astronomical Institute, M. V. Lomonosov Moscow State University, Universitetskij Prosp. 13, Moscow 119991, Russia; mike.prokhorov@gmail.com

3 South African Astronomical Observatory, PO Box 9, Observatory, Cape Town 7935, South Africa; akniazev@saao.ac.za

4 South African Large Telescope Foundation, PO Box 9, Observatory, Cape Town 7935, South Africa

Received: 2015 March 25; accepted: 2015 April 20

\begin{abstract}
The principal goal of this paper is to specify conditions of detection of unresolved binaries by multicolor photometry. We have developed a method for estimating the critical distance at which an unresolved binary of given mass and age can be detected. The method is applied to the photometric system of the planned Lyra-B spaceborne experiment. We have shown that some types of unresolved binary stars can be discovered and distinguished from single stars solely by means of photometric observations.
\end{abstract}

Key words: binaries: general - techniques: photometric

\section{INTRODUCTION}

Binary stars are very numerous, however only the nearest pairs can be resolved into components. Others remain unresolved, and the vast majority of them are observed neither as spectroscopic nor as eclipsing binaries. On the other hand, the effect of unresolved binaries is extremely important for the construction of the luminosity function (Piskunov \& Malkov 1991; Kroupa et al. 1991) and the initial mass function (Malkov 2002). Therefore, the detection and analysis of photometrically unresolved binaries remain a vital and promising avenue of investigation.

If both components of a binary star have relatively different effective temperatures, but comparable luminosities, the color indices of such a system will differ from those of a single star. Generally speaking, even a binary with identical components can be distinguished from single stars, because it is located on the HR diagram above the position of the corresponding single star. However, in many cases we do not have reliable estimates of stellar distances, therefore it is usually impossible to distinguish between a binary and a single star solely by means of one-band photometry.

The problem of detection and classification of unresolved binaries by multicolor 


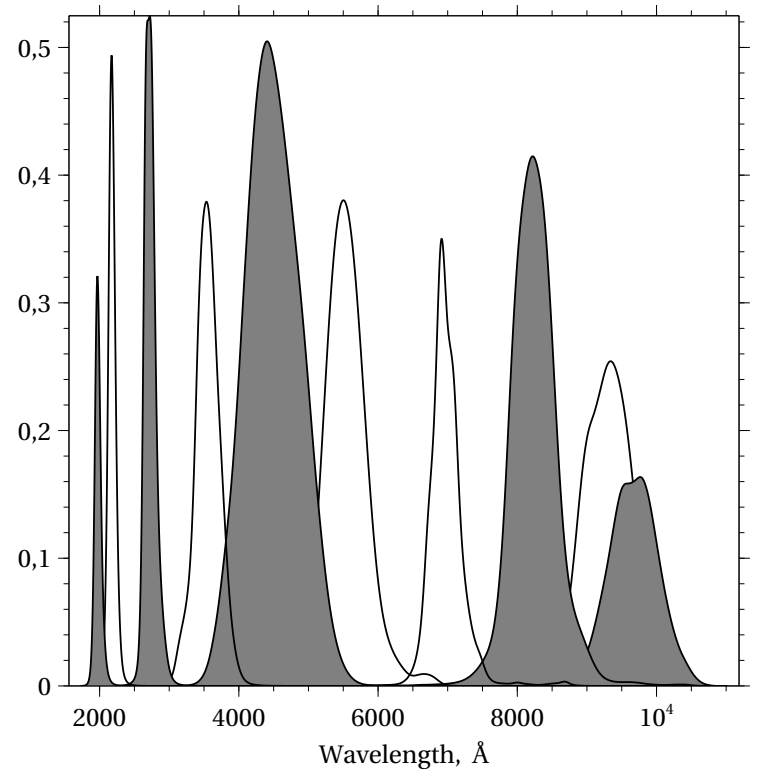

Fig. 1. Response curves of the Lyra-B photometric system. Transmission of the interference filters and CCD quantum efficiency are taken into account.

photometry was studied for the Gaia (Malkov et al. 2011a) and ultraviolet (Malkov et al. 2011b) photometric systems. Also, various methods were proposed for the identification of unresolved binaries among very low mass stars and brown dwarfs by spectroscopic observations (Bardalez Gagliuffi et al. 2014) or by SDSS/2MASS cross-matching (Geissler et al. 2011). In the current paper we develop a new method for binary detection, estimate the critical distance at which binary stars can be discovered, and apply the method to the Lyra-B photometric system.

Lyra-B (Zakharov et al. 2013a) is a space-based experiment onboard the International Space Station, which is currently being prepared at the Sternberg Astronomical Institute. The main goal of the experiment is to carry out a highaccuracy, multicolor all-sky survey of stars down to 16-17 mag. The response curves of the Lyra-B ten-band photometric system are shown in Fig. 1, and the central wavelengths of the bands are 195, 218, 270,350,440, 555, 700, 825, 930, $1000 \mathrm{~nm}$. During the Lyra-B mission, an all-sky high-precision photometric survey of objects from 3 to $16 \mathrm{mag}$ will be undertaken. The photometric errors of the survey catalog are expected to be about $0.001-0.003 \mathrm{mag}$ for stars brighter than 12 mag and about 0.01 mag for fainter stars. The estimated duration of the survey is about five years. The Lyra-B photometric system is described in detail by Zakharov et al. (2013b), while the expected characteristics of output data of the mission can be found in Zakharov et al. (2013c).

The proposed method for the detection of unresolved binaries is described in Section 2. The application of the method to the Lyra-B photometric system is illustrated in Section 3. In Section 4 we draw our conclusions. 


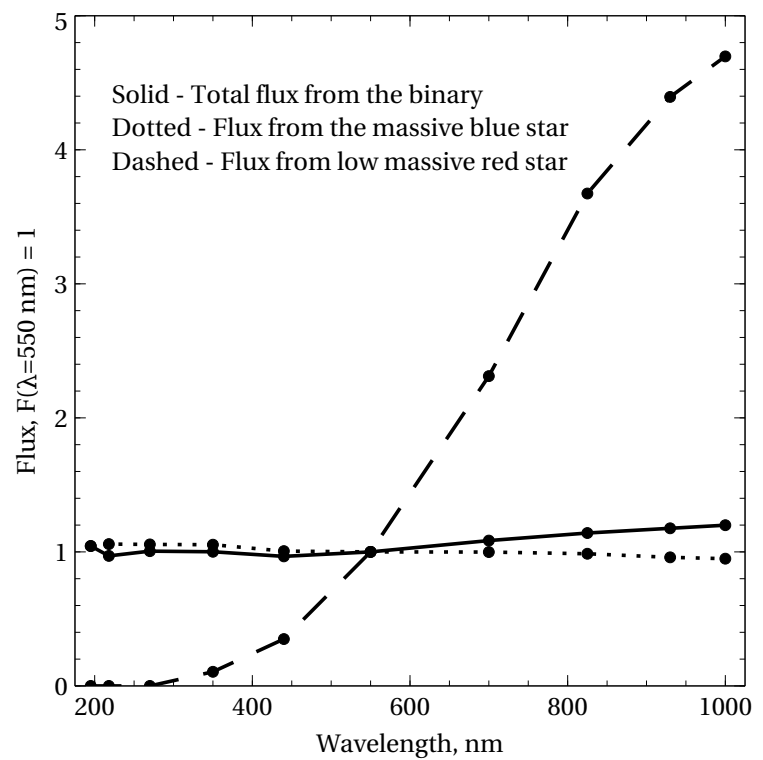

Fig. 2. Example of spectral energy distribution (SED) for a binary star. The dashed and dotted lines are SEDs of the components, while the solid line represents the total flux.

\section{DESCRIPTION OF THE METHOD}

Theoretical color indices of binary stars can be obtained from tracks and isochrones of single stars (this provides us with stellar astrophysical parameters: bolometric luminosity $L$, effective temperature $T_{\text {eff }}$ and surface gravity $g$, for given mass $M$, age $\tau$ and metallicity) and with grids of model atmospheres (which simulate observational photometry for a star with given astrophysical parameters). Then, the simulated fluxes, when combined with the photometric system's response curves, provide us with theoretical color-indices. The problem of the accuracy of astrophysical stellar parameters determined from multicolor photometry was studied by Sichevskij et al. (2014).

To generate isochrones, we used PARSEC stellar evolution code (Bressan et al. 2012 ) for the age $\tau=10^{8}$ years and solar metallicity. The astrophysical parameters $L, T_{\text {eff }}$ and $g$ were obtained for stellar masses in the range $0.1-5.3 M_{\odot}$, with a step of $0.1 M_{\odot}$. The upper mass limit is determined by the age value $\left(\tau=10^{8} \mathrm{yr}\right)$. We used a linear approximation to compute the astrophysical parameters for a star of a particular mass.

To get the fluxes for single stars we then used grids of ATLAS9 model atmospheres (Castelli \& Kurucz 2003) for $T_{\text {eff }}, \log g$ and interstellar extinction $A_{V}$, calculated with steps of $250 \mathrm{~K}, 0.25 \mathrm{dex}$ and $0.25 \mathrm{mag}$, respectively. Throughout the paper, we adopt the total to selective extinction ratio $R_{V}=3.1$. We approximate the flux of any given intermediate point $\left(T_{\text {eff }}, \log g, A_{V}\right)$ within the local axial rectangular prism by trilinear interpolation, using data at the eight corner points (reference stars with known fluxes) on the cube surrounding the interpolation point. The normalized flux is considered to be $F(\lambda=550 \mathrm{~nm})=1$.

The fluxes of two components are combined in accordance with their luminosity 
ratio (see Fig. 2), and the color indices are calculated for given response curves. We assume extinction $A_{V}$ to be the same for both components. The resulting color indices of the binary star are compared with those of single stars from the atlas, for various $A_{V}$ values. The best-fit single star is selected via the $\chi^{2}$ minimization procedure:

$$
\chi_{0}^{2}=\sum_{k}^{1 . .10} \frac{\left(f_{\mathrm{b}}(k)-f_{\mathrm{s}}(k)\right)^{2}}{f_{\mathrm{b}}(k)},
$$

where $k$ is spectral band number, $f_{\mathrm{b}}(k)$ and $f_{\mathrm{s}}(k)$ are the binary and single comparison star fluxes in the given band $k$, respectively.

The normalized fluxes are considered to be $f_{\mathrm{s}}(\lambda=550)=f_{\mathrm{b}}(\lambda=550)=1$. For a real star, $f_{\mathrm{b}}(\lambda=550) \neq 1$, and $\chi_{\text {obs }}^{2}=\chi_{0}^{2} \cdot N(\lambda=550)$. Thus we find a critical distance $d_{\text {crit }}$, for which $\chi_{\text {obs }}^{2}$ equals $\chi_{\text {crit }}^{2}$. Critical $\chi_{\text {crit }}^{2}$ is selected manually, and in our calculations we assumed that $\chi_{\text {crit }}^{2}=27$, which corresponds to a confidence level of more than $99 \%$.

Note that in this paper we refer exclusively to non-interacting binaries.

\section{RESULTS}

Hereafter, we consider non-reddened binary systems. Our estimates show that for reddened systems, at least up to $A_{V}=2 \mathrm{mag}$, the results are qualitatively the same, though all the resulting values of $d_{\text {crit }}$ should be decreased. However, we study the effect of reddening of comparison single stars. In most of the cases considered, the best-fit single star is not reddened. Interstellar reddening can be large enough for well evolved massive primaries ( $A_{V}$ up to $2.7 \mathrm{mag}$ ), but for main-sequence stars the largest reddening, $A_{V}=0.44 \mathrm{mag}$, is achieved just for the $0.7 M_{\odot}+0.4 M_{\odot}$ system. In the latter case, the single comparison star is hotter $(4600 \mathrm{~K})$ than both the primary $(4500 \mathrm{~K})$ and the secondary $(3900 \mathrm{~K})$. The temperature of the comparison star is usually intermediate between those of the two components.

Fig. 3 shows the critical distances estimated for the isochrone $\tau=10^{8}$ years. For further analysis we fix the primary mass $\left(M_{1}\right)$ and estimate the critical distance as a function of the mass of the secondary. Fig. 4 shows the results for $M_{1}=1$, $2,3,4$ and $5 M_{\odot}$.

Because of the low luminosity of subsolar-mass binaries, their critical distance does not exceed $d_{\text {crit }} \approx 35$ pc. Pairs with low mass ratios $q=M_{2} / M_{1}$ tend to have better chances to be detected as binaries.

The distribution curves for the primary masses 2 and $3 M_{\odot}$ show a well pronounced single peak. Stars with similar masses have very similar spectra, making it hard to detect their binarity from multicolor photometry. On the other hand, pairs with a low-mass companion contribute negligibly to the part of the distribution corresponding to distinctly red secondaries because of the dramatically low luminosity of the former. These two phenomena produce a steep peak. According to our calculations, the maximum critical distance is achieved for a $2.3 M_{\odot}+1.5 M_{\odot}$ binary star, which can be detected out to a distance of $500 \mathrm{pc}$.

For more massive primaries, the peak is less pronounced, and turns into a plateau. In particular, for a $4 M_{\odot}$ primary, the distribution shows two extrema, at the secondary masses $1.5 M_{\odot}$ and $2.7 M_{\odot}$. This can be explained by a greater 


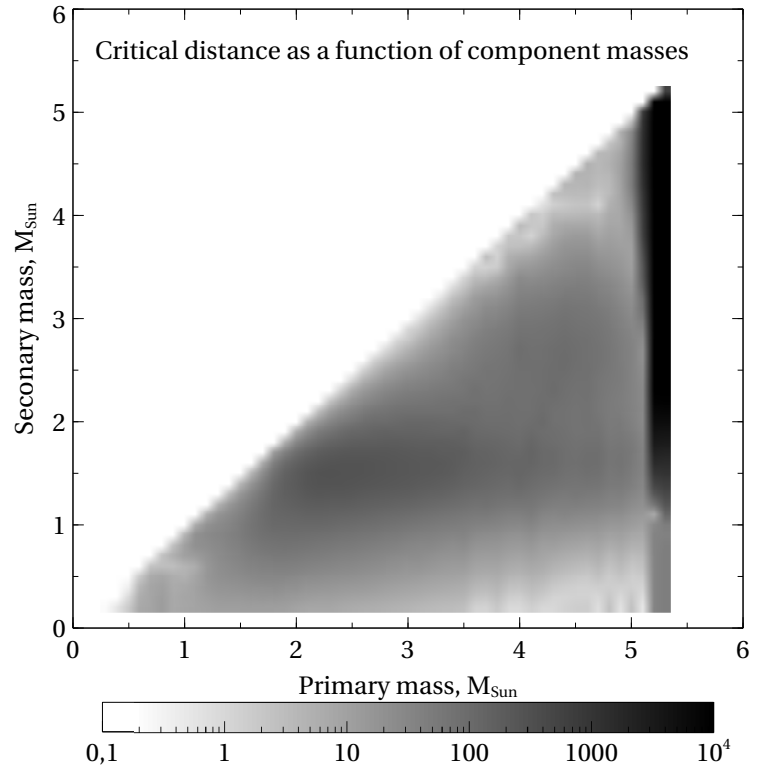

Fig. 3. Critical distance of binary detection as a function of the component masses. The darker shading corresponds to larger distances.

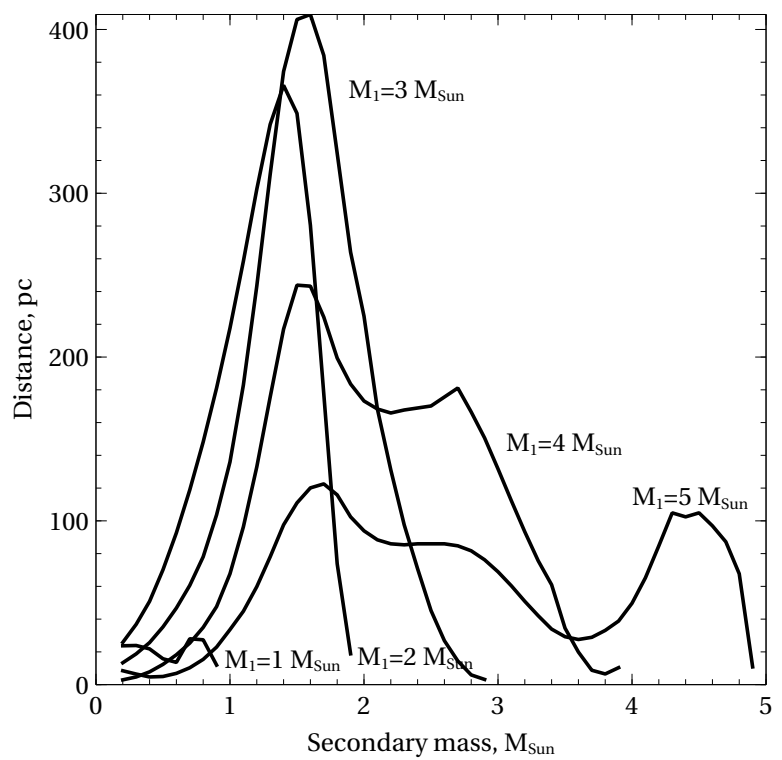

Fig. 4. Critical distance of binary detection as a function of the secondary mass for primary masses $M_{1}=1,2,3,4$ and $5 M_{\odot}$. 


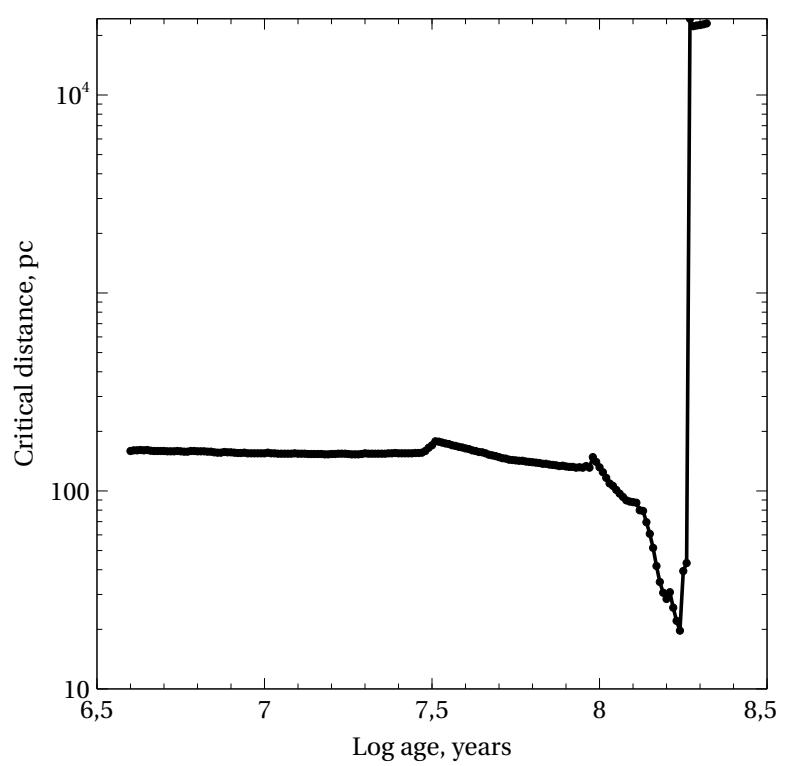

Fig. 5. Critical distance of binary detection as a function of age for the system with a $4 M_{\odot}$ primary and a $3 M_{\odot}$ secondary.

variety of single stars used for the comparison. Consequently, it is much easier to find a single star emulating a particular binary, and this causes some drop in the critical distance (as it can be seen in Fig. 4). The maximum critical distance drops down to 325 and $175 \mathrm{pc}$ for the $3.5 M_{\odot}$ and $4.5 M_{\odot}$ primary, respectively.

The calculations discussed above are obtained for a single isochrone, and we therefore need to investigate the evolutionary effects. Because the calculations are time consuming we focus only on a particular binary and calculate its detectability throughout the lifetime. The binary considered consists of a $4 M_{\odot}$ primary and a $3 M_{\odot}$ secondary. Given an upper limit for the lifetime of the primary, we performed our calculations for the ages ranging from $\log \tau=6.6$ (around 4 million years) to $\log \tau=8.32$ (around 200 million years), with a step of $\log \tau=0.01$. The results are shown in Fig. 5.

Initially, the primary star has a higher temperature and luminosity than its companion. In the process of stellar evolution the luminosities of both components gradually increase and their effective temperatures decrease. While on the main sequence, this process is slow and has no significant effect on the observed color indices of the system. This means that our above predictions for a certain isochrone apply to the entire main-sequence stage. This period lasts until about $\log \tau \approx 8.0$ (100 million years) for the binary considered. Of course, it would last much longer for less massive and more numerous solar-mass primaries.

After the primary component leaves the main sequence, its evolution speeds up and evolutionary processes occur on a shorter timescale. The temperature decreases more rapidly and, at some point (around $\log \tau \approx 8.23$ in our case), it reaches that of the less massive companion, which is still on the main sequence. This makes the binary detection much more difficult, and the critical distance reaches its minimum value. Finally, the primary reaches the Asymptotic Giant 
Branch, and we are left with a system of markedly different (bright red primary and blue secondary) components. The difference of the effective temperatures becomes by far the largest during the lifetime of the system, enabling the best conditions for multicolor observations.

\section{CONCLUSIONS}

We developed a method for detecting unresolved binaries based on multicolor photometry. We apply the method to the ten-band photometric system of the planned Lyra-B mission. We estimate the critical distance for the detection of a binary as a function of its age and component masses. Our method can also be extrapolated to higher masses and can also be applied for other photometric systems.

ACKNOWLEDGMENTS. The work was partly supported by the Program of fundamental research of the Presidium of the Russian Academy of Sciences (P41), the Russian Foundation for Basic Research (project No. 15-02-04053), and the Program of support of leading scientific schools of the Russian Federation (3620.2014.2). A. K. acknowledges support from the National Research Foundation of South Africa.

\section{REFERENCES}

Bardalez Gagliuffi D. C., Burgasser A. J., Gelino Ch. R. 2014, ApJ, 794, 143

Geissler K., Metchev S., Kirkpatrick J. D. 2011, ApJ, 732, 56

Bressan A., Marigo P., Girardi L. et al. 2012, MNRAS, 427, 127

Castelli F., Kurucz R. L. 2003, in Modelling of Stellar Atmospheres (IAU Symp. 210), eds. N. Piskunov, W. W. Weiss \& D. F. Gray, ASP, San Francisco, p. A20

Kroupa P., Gilmore G., Tout Ch. A. 1991, MNRAS, 251, 293

Malkov O. 2002, Ap\&SS, 280, 129

Malkov O. Yu., Mironov A. V., Sichevskij S. G. 2011a, EAS Pub. Ser. 45, 409

Malkov O. Yu., Mironov A. V., Sichevskij S. G. 2011b, Ap\&SS, 335, 105

Piskunov A. E., Malkov O. Yu. 1991, A\&A, 247, 87

Sichevskij S. G., Mironov A. V., Malkov O. Yu. 2014, Astrophysical Bulletin, 69, 160

Zakharov A. I., Mironov A. V., Prokhorov M. E. et al. 2013a, Astronomy Reports, 57,195

Zakharov A. I., Mironov A. V., Nikolaev F. N. et al. 2013b, AN, 334, 823

Zakharov A. I., Mironov A. V., Prokhorov M. E. et al. 2013c, AN, 334, 828 